\title{
Cooperative Sensing Decision Rules over Imperfect Reporting Channels
}

\author{
Nian Xia ${ }^{1, a}$, Chu-Sing Yang ${ }^{1, b}$ \\ ${ }^{1}$ Institute of Computer and Communication Engineering, Department of Electrical Engineering, \\ National Cheng Kung University, Tainan, 70101, Taiwan \\ aemail: brastme@gmail.com, bemail: csyang@ee.ncku.edu.tw
}

Keywords: cooperative spectrum sensing; hard decisions; Selection-based MRC; imperfect reporting channels

\begin{abstract}
Cognitive Radio (CR) is proposed to solve the spectrum scarcity problem caused by the explosive growth of wireless devices and the fixed spectrum allocation policy. Spectrum Sensing is a fundamental method in CR. Cooperative Spectrum Sensing (CSS) can effectively improve detection accuracy. Numerous works considering perfect reporting channels exist. However, imperfect reporting channels have not gained enough attention until now. In this paper, we investigate imperfect flat Rayleigh fading listening and reporting channels. We use channel state information to assist Fusion Center (FC) to make final decisions, and propose several channel-information-based MRC decision rules. Finally, traditional hard decision rules are compared with our proposed channel-information-based MRC decision rules. Our Selection-based MRC decision rule can achieve the lowest total error rate.
\end{abstract}

\section{Introduction}

CR was initially proposed by Mitola [1] in 1999. Simon Haykin [2] defined CR as: "an intelligent wireless communication system that is aware of its environment and uses the methodology understanding-by-building to learn from the environment and adapt to statistical variations in the stimuli".

Dynamic Spectrum Access (DSA) allows secondary users (SUs) to use licensed spectrum resources when primary users (PUs) are idle or the interference to PUs is below a constraint [3]. Spectrum Sensing (SS) is used to detect the presence of PU. There are three major spectrum sensing detectors [4-6]. Energy detector (ED) is a non-coherent detector which detects PU based on the sensed energy. ED is widely used due to its simplicity and no need prior knowledge of PU. However, ED does not perform very well in low SNR regions. Matched filter detector is a linear detector which can maximize the output signal-to-noise ratio (SNR), but prior knowledge of PU is needed. Cyclostationary detector uses the periodicity in the received signal to determine PU's state, and it is robust to noise uncertainty. Cooperative Spectrum Sensing (CSS) is proved to be able to effectively improve the spectrum sensing detection performance by using spatial diversity. Different SUs independently sense licensed channels and forward their decisions to FC via reporting channels. Different decision rules are employed to decide whether PU is idle or not [7, 8].

Decision rules in the receiver have gained enough attentions recently. E. Axell et al. investigate hard decision rules which use one bit to represent PU's activity. They compare AND, OR and Voting rules under different listening channel conditions. The Voting rule is regarded to perform best among the three hard decision rules [9]. Literatures above all assume that reporting channel is error-free. Data transmissions from SUs to FC are not error free due to multipath, noise, shadowing and so on. Sachin Chaudhari et al. $[10,11]$ prove that Bit Error Probability (BEP) wall exists over imperfect reporting channels in different combing rules. Soft decision rules gain lower BEP in the cost of channel bandwidth to transmit more than one bit information. However, they assume that reporting channel is Binary Symmetric Channel (BSC) which is comparatively not realistic. In their recent works, they generalize reporting channels and prove that BEP wall still exists over Rayleigh fading reporting channels [12]. To simplify mathematical derivations, they assume that listening channel follows Gaussian distribution. Furthermore, they do not use channel information which can 
be easily obtained by using estimation theory [13]to assist FC to make final decisions.

In this paper, we consider Cognitive Radio Networks (CRNs) with one FC. We assume that both listening channels and reporting channels follow Rayleigh distributions. Common Control Channels (CCC) are used to exchange information between SUs and FC. We compare several channel-information-based MRC (Maximize Ration Combing) decision rules, and propose a novel Selection-based MRC decision rule. This paper's contributions are summarized as follows:

- Investigate imperfect Rayleigh fading reporting channel.

- Propose and compare several channel-information-based MRC decision rules.

- Propose a novel Selection-based MRC decision rule.

\section{Basic Principles}

\section{Cooperative sensing system model}

We consider a cooperative sensing system containing a PU transmitter, $N$ SUs and a FC in Fig. 1. Both listening channels and reporting channels are independent and identically distributed (i.i.d.). ED is employed in each SU to detect PUs. One bit hard decision is then forwarded by SUs to FC. FC then uses different decision rules to make a final decision.

We define $I$ as an indicator function. SUs compare received signal's energy $E$ with predetermined energy detector threshold $\gamma .1$ indicates that PU is using the channel, and 0 indicates that $\mathrm{PU}$ is absent. We express $I$ as follows:

$$
I= \begin{cases}1, & E \geq \gamma \\ 0, & E<\gamma\end{cases}
$$

$s(t)$ denotes that SU transmits equivalent low-pass binary decision. $h$ and $\theta$ represent the attenuation factor and phase shift for the channel respectively. $n(t)$ denotes Addition white Gaussian noise (AWGN). The received low-pass signal can be written as follows:

$$
r(t)=h e^{j \theta} s(t)+n(t) \quad 0 \leq t \leq T .
$$

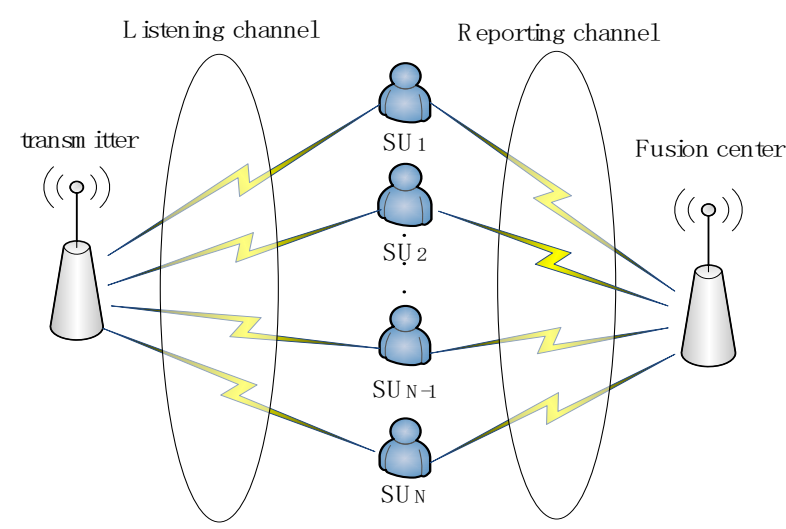

Fig. 1. The wireless communication system where listening channel and reporting channel both follow Rayleigh distribution.

\section{False alarm probability and miss detection probability}

$H 0$ and $H 1$ denote that PU is idle and busy respectively. $P_{f}=\operatorname{Pr}(I=1 \mid H 0)$, also known as false alarm probability, denotes that we decide PU is present while PU is actually absent. Similarly, $P_{m}=\operatorname{Pr}(I=0 \mid H 1)$, termed as miss detection probability, denotes that we decide PU is absent while PU is actually occupying the channel. We denote the $i$-th SU's detection probability as follows:

$$
P_{d, i}=1-P_{m, i} \text {. }
$$

In (3), $P_{m, i}$ denotes the $i$-th SU's miss detection probability. We assume that every SU has the 
same energy detector threshold $\gamma$, so every SU has the same detection probability:

$$
P_{d}=P_{d, i}, i=1,2, \ldots, N \text {. }
$$

The $i$-th SU's total error rate can be shown as follows:

$$
P_{\text {total }, i}=P_{f, i}+P_{m, i} \text {. }
$$

Suitable energy detector threshold $\gamma$ is obtained to decrease FC's total error rate. Similarly, we express FC's total error rate as follows:

$$
P_{\text {total }}^{\text {rule }}=P_{f}^{\text {rule }}+P_{m}^{\text {rule }} .
$$

In (6), $P_{f}^{\text {rule }}, P_{m}^{\text {rule }}$ and rule respectively denote false alarm probability, miss detection probability and different decision rules.

\section{Decision Rules}

\section{Existing decision rules}

In perfect reporting channels, several hard decision rules including AND rule, OR rule and Voting rule have been discussed. Voting rule can achieve the lowest total error rate. We briefly introduce the basic schemes of the above three hard decision rules in turn.

AND rule: PU is said to be present only when all SUs decide that PU is present. The detection probability, false alarm probability, and miss detection probability in the FC are written as follows:

$$
\begin{aligned}
& P_{d}^{\text {and }}=\prod_{i=1,2, \ldots, N} P_{d, i} . \\
& P_{f}^{\text {and }}=\prod_{i=1,2, \ldots, N} P_{f, i} . \\
& P_{m}^{\text {and }}=1-\prod_{i=1,2, \ldots, N} P_{d, i} .
\end{aligned}
$$

OR rule: FC decides that PU is present when at least one SU declares that PU is present. Similarly, the probabilities for the OR rule are shown below:

$$
\begin{aligned}
& P_{d}^{o r}=1-\prod_{i=1,2, \ldots, N}\left(1-P_{d, i}\right) . \\
& P_{f}^{o r}=1-\prod_{i=1,2, \ldots, N}\left(1-P_{f, i}\right) . \\
& P_{m}^{o r}=\prod_{i=1,2, \ldots, N}\left(1-P_{d, i}\right) .
\end{aligned}
$$

Voting rule: FC decides that PU is present if more than half SUs decide that PU is present. The probabilities for the voting rule in the FC are shown as follows:

$$
\begin{aligned}
& P_{d}^{\text {major }}=\sum_{k=[N / 2\rfloor}^{N}\left(\begin{array}{l}
N \\
k
\end{array}\right) P_{d}^{k}\left(1-P_{d}\right)^{N-k} . \\
& P_{f}^{\text {major }}=\sum_{k=[N / 2\rfloor}^{N}\left(\begin{array}{l}
N \\
k
\end{array}\right) P_{f}^{k}\left(1-P_{f}\right)^{N-k} . \\
& P_{m}^{\text {major }}=1-\sum_{k=\lfloor N / 2\rfloor}^{N}\left(\begin{array}{l}
N \\
k
\end{array}\right) P_{d}^{k}\left(1-P_{d}\right)^{N-k} .
\end{aligned}
$$

\section{EGC-based decision rule and MRC-based decision rule}

Considering imperfect reporting channels, FC can combine different SUs' transmitted signals with corresponding channel state information (CSI) by using different combing rules to make final decisions. Assume $N$ different reporting channels with equal noise power, we can express the FC combiner output as follows:

$$
d_{f c}=\sum_{i=1}^{N} w_{i} e^{-j \theta_{i}} r_{i}
$$


In (16), $w_{i}$ denotes the weight of $i$-th SU's decision and $\sum_{i=1}^{N} w_{i}=1$, the complex exponential is used for equalizing the phase of each reporting channel, $r_{i}$ denotes the received signal from $i$-th SU's local decision as defined in (2).

EGC combing rule: Every SU has the same weight, and then $w_{i}$ equals to $1 / N$. Below we introduce three channel-information-based MRC decision rules. First, we define some necessary notations. $h_{l s, i}$ and $h_{r e, i}$ denote fading amplitudes for the i-th SU's listening channel and reporting channel respectively. We denote the multiplication of listening channel and reporting channel of the i-th SU as follows:

$$
h_{\text {prod }, i}=h_{l s, i} * h_{r e, i} .
$$

In the Listen-based MRC, Report-based MRC and Channel-based MRC, FC uses listening channel state information, reporting channel information and the multiplication of listening and reporting channels to do MRC respectively. The $i$-th SU's weight $w_{\text {mod,i }}$ is written as follows:

$$
w_{\bmod , i}=\frac{h_{\bmod , i}}{\sum_{i=1}^{N} h_{\bmod , i}} .
$$

Where $h_{\text {mod, }, i}$ is set to $h_{l s, i}, h_{r e, i}$ and $h_{p r o d, i}$ respectively in different MRC rules.

\section{Selection-based MRC}

In our assumptions, the channel link gain between FC and SUs is comparatively higher than the channel link gain between PU transmitter and SUs. Therefore, listening channel is more important than reporting channel. To reduce SU's energy consumption and decrease system's total error rate, we choose SUs with better listening channel qualities to coordinate.

$$
w_{i}=\frac{h_{l s, i}}{\sum_{\substack{i=1 \\ \text { s.t. } h_{l, i} \geq \lambda}}^{N} h_{l s, i}}
$$

$\lambda$ denotes the listening channel quality threshold. We will discuss more about the threshold $\lambda$ in the next section.

\section{Simulation Results}

\section{Experimental environments}

In our experiment, listening channels and reporting channels follow Rayleigh distribution. Noise is AWGN with mean zero and variance one. We employ the BPSK modulation in both the PU transmitter and SUs. Carrier Frequency is set to be $800 \mathrm{MHz}$ and bandwidth is $2 \mathrm{MHz}$. Reporting channel SNR is $25 \mathrm{~dB}$. We simulate more than 10000 times.

\section{Simulation results}

Firstly, we investigate the relationship between EGC, Channel-information-based MRC decision rules' listening channel threshold $\lambda$ and system's total error rate. We assume that the listening channel SNR is $0 \mathrm{~dB}$ and the reporting channel SNR is $25 \mathrm{~dB}$. We vary the listening channel threshold $\lambda$ from 0 to 3 and observe the corresponding total error rate.

In Fig. 2., among compared five decision rules, only Selection-based MRC will choose SU's with better listening channel condition to coordinate, so only its total error rate varies with different listening threshold $\lambda$. Suboptimal listening channel threshold $\lambda$ exists with the varying listening $\lambda$ channel values. With the increasing listening channel threshold, the sensing accuracy will increase; however, the number of selected SUs will decrease. System's total error rate will increase because we can't select enough SUs to coordinate. There is a tradeoff between threshold $\lambda$ and the number of selected SUs. Listen-based MRC achieves the second lowest total error rate due to the fact that listening channel has a higher priority than reporting channel. Similarly, we can explain why 
Report-based MRC has the highest total error rate. In our proposed Selection-based MRC, SUs with listening channel's parameter equal or larger than threshold $\lambda$ are selected to assist FC making final decisions. By choosing suitable threshold $\lambda$, Selection-based MRC can achieve the lowest total error rate.

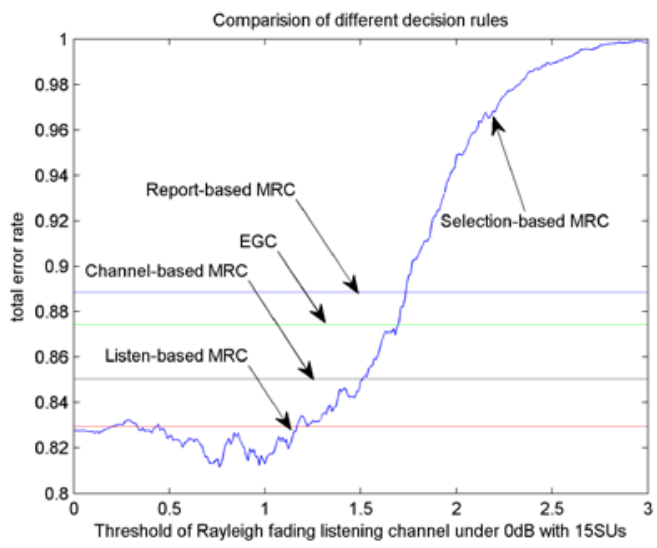

Fig. 2. The relationship of listening channel thresholds with total error rate.

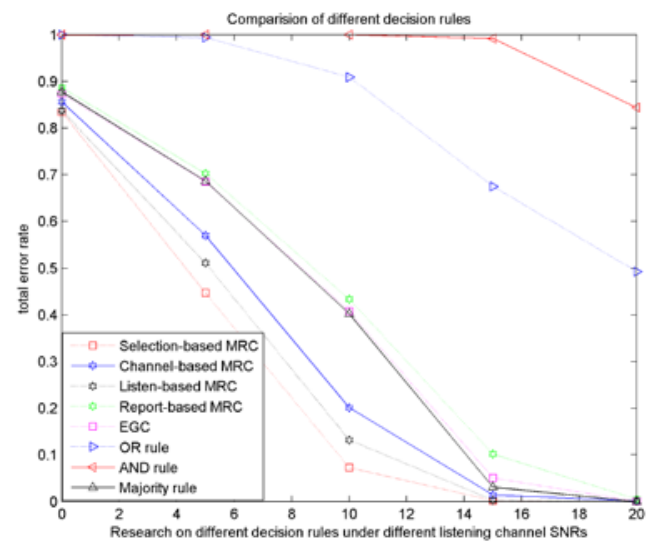

Fig. 3. Compare different decision rules in different listening channel SNRs.

In Fig. 3., our proposed Selection-based MRC has the lowest total error rate among different decision rules in different SNR situations. However, in low SNR regions, due to the limited number of cooperative SUs, we may not find suitable SUs to coordinate. So it is reasonable that our proposed Selection-based MRC does not always achieve the lowest total error rate in low SNR regions. Meanwhile, our proposed channel-information-based MRC decision rules perform better than traditional hard decision rules.

\section{Conclusion}

In this paper, we investigate imperfect Rayleigh fading reporting channels and propose several channel-information based MRC decision rules: Listen-based MRC, Report-based MRC, and Channel-based MRC decision rules. Furthermore, we propose a novel Selection-based MRC decision rule which chooses SUs with better listening channel quality to coordinate. Finally, we compare our proposed scheme with traditional hard decision rules. Our Selection-based MRC decision rule can achieve the lowest total error rate.

In our future work, we will consider SUs with different spatial locations, and SUs can adapt their transmission power to save energy. In addition, this proposed scheme needs to be comprehensively evaluated when the channel estimation error is considered. Theoretic analysis is required to prove our simulation results. Furthermore, we will investigate the decision rule performance when malicious SUs exist. 


\section{Acknowledgement}

This work was supported in part by Taiwan Ministry of Science and Technology (MOST) under Grant MOST 104-3115-E-194-001.

\section{References}

[1] J. Mitola III and G. Q. Maguire, "Cognitive radio: making software radios more personal," IEEE Personal Communications, vol. 6, no. 4, pp. 13-18, Aug. 1999.

[2] S. Haykin, "Cognitive radio: brain-empowered wireless communications," IEEE Journal on Selected Areas in Communications, vol. 23, no. 2, pp. 201-220, Feb. 2005.

[3] Z. Qing and B. M. Sadler, "A Survey of Dynamic Spectrum Access," IEEE Signal Processing Magazine, vol. 24, no.3, pp. 79-89, May 2007.

[4] P. D. Sutton, J. Lotze, K. E. Nolan, and L. E. Doyle, "Cyclostationary Signature Detection in Multipath Rayleigh Fading Environments," 2nd International conference on Cognitive Radio Oriented Wireless Networks and Communications, 2007.

[5] T. Yucek and H. Arslan, "A survey of spectrum sensing algorithms for cognitive radio applications," IEEE Communications Surveys and Tutorials, vol. 11, no. 1, pp. 116-130, 2009.

[6] A. Sahai, N. Hoven, and R. Tandra, "Some Fundamental Limits on Cognitive Radio," 42sd Allerton Conference on Communication, Control and Computing, 2004.

[7] I. F. Akyildiz, B. F. Lo, and R. Balakrishnan, "Cooperative spectrum sensing in cognitive radio networks: A survey," Physical Communications, vol. 4, no. 1, pp. 40-62, 2011.

[8] S. Nallagonda, S. D. Roy, and S. Kundu, "Cooperative spectrum sensing with censoring of cognitive radios in Rayleigh fading channel," 2012 National Conference on Communications (NCC), 2012.

[9] E. Axell, G. Leus, and E. G. Larsson, "Overview of spectrum sensing for cognitive radio," 2nd International Workshop on Cognitive Information Processing (CIP), 2010.

[10] S. Chaudhari, J. Lunden, V. Koivunen, and H. V. Poor, "Cooperative Sensing With Imperfect Reporting Channels: Hard Decisions or Soft Decisions?," IEEE Transaction on Signal Processing, vol. 60, no. 1 pp. 18-28, Jan. 2012.

[11] S. Chaudhari, J. Lunden, and V. Koivunen, "BEP walls for collaborative spectrum sensing," 36th IEEE International Conference on Acoustics, Speech and Signal Processing, 2011.

[12] S. Chaudhari, J. Lundén, V. Koivunen, and H. V. Poor, "BEP walls for cooperative sensing in cognitive radios using K-out-of-N fusion rules," Signal Processing, vol. 93, pp. 1900-1908, 2013.

[13] S. M. Kay, "Fundamentals of Statistical Signal Processing: Estimation Theory," Prentice Hall, 1993. 